\title{
Nitric oxide maintains cell survival of Trichomonas vaginalis upon iron depletion
}

\author{
Wei-Hung Cheng 1,2, Kuo-Yang Huang 2,3, Po-Jung Huang ${ }^{3,4}$, Jo-Hsuan Hsu' ${ }^{1,2}$, Yi-Kai Fang ${ }^{1,2}$, Cheng-Hsun Chiu ${ }^{5}$
} and Petrus Tang ${ }^{1,2,45^{*}}$

\begin{abstract}
Background: Iron plays a pivotal role in the pathogenesis of Trichomonas vaginalis, the causative agent of highly prevalent human trichomoniasis. T. vaginalis resides in the vaginal region, where the iron concentration is constantly changing. Hence, T. vaginalis must adapt to variations in iron availability to establish and maintain an infection. The free radical signaling molecules reactive oxygen species (ROS) and reactive nitrogen species (RNS) have been proven to participate in iron deficiency in eukaryotes. However, little is known about the roles of these molecules in iron-deficient $T$. vaginalis.
\end{abstract}

Methods: T. vaginalis cultured in iron-rich and -deficient conditions were collected for all experiments in this study. Next generation RNA sequencing was conducted to investigate the impact of iron on transcriptome of $T$. vaginalis. The cell viabilities were monitored after the trophozoites treated with the inhibitors of nitric oxide (NO) synthase (L-NG-monomethyl arginine, L-NMMA) and proteasome (MG132). Hydrogenosomal membrane potential was measured using JC-1 staining.

Results: We demonstrated that NO rather than ROS accumulates in iron-deficient $T$. vaginalis. The level of NO was blocked by MG132 and L-NMMA, indicating that NO production is through a proteasome and arginine dependent pathway. We found that the inhibition of proteasome activity shortened the survival of iron-deficient cells compared with untreated iron-deficient cells. Surprisingly, the addition of arginine restored both NO level and the survival of proteasome-inhibited cells, suggesting that proteasome-derived NO is crucial for cell survival under iron-limited conditions. Additionally, NO maintains the hydrogenosomal membrane potential, a determinant for cell survival, emphasizing the cytoprotective effect of $\mathrm{NO}$ on iron-deficient $\mathrm{T}$. vaginalis. Collectively, we determined that NO produced by the proteasome prolonged the survival of iron-deficient T. vaginalis via maintenance of the hydrogenosomal functions.

Conclusion: The findings in this study provide a novel role of NO in adaptation to iron-deficient stress in $T$. vaginalis and shed light on a potential therapeutic strategy for trichomoniasis.

Keywords: Iron-deficiency, Nitric oxide, Arginine, Ubiquitin-proteasome system, Hydrogenosomal membrane potential, Cell survival

\footnotetext{
* Correspondence: petang@mail.cgu.edu.tw

${ }^{1}$ Graduate Institute of Biomedical Sciences, College of Medicine, Chang Gung University, Kweishan, Taoyuan, Taiwan

${ }^{2}$ Molecular Regulation and Bioinformatics Laboratory, Department of Parasitology, College of Medicine, Chang Gung University, Kweishan, Taoyuan, Taiwan

Full list of author information is available at the end of the article
}

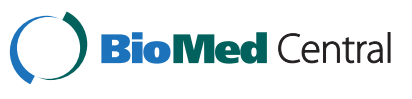

(C) 2015 Cheng et al. This is an Open Access article distributed under the terms of the Creative Commons Attribution License (http://creativecommons.org/licenses/by/4.0), which permits unrestricted use, distribution, and reproduction in any medium, provided the original work is properly credited. The Creative Commons Public Domain Dedication waiver (http:// creativecommons.org/publicdomain/zero/1.0/) applies to the data made available in this article, unless otherwise stated. 


\section{Background}

Trichomonas vaginalis is a unicellular pathogen that causes human trichomoniasis, one of the most prevalent sexually transmitted diseases worldwide [1]. Iron deficiency in the host affects several biological processes in T. vaginalis, including cell proliferation, cytotoxicity and immune evasion [2-4]. Iron-containing proteins, such as lactoferrin and hemoglobin, can be used by $T$. vaginalis [5], and these proteins are mainly supplied by menstrual blood [6]. The constant change in environmental iron availability might be the major challenge for the protist to survive in the vaginal region [5]. Therefore, the protist must adapt to an environment with different iron concentrations to establish or maintain an infection. The iron level has to be tightly controlled because overload or deficiency can cause cellular damages [7, 8]. However, the mechanisms that help T.vaginalis cope with iron stresses remain poorly understood.

Redox homeostasis is an important issue for cellular functions because excessive free radicals destroy biomolecules [9]. A previous study demonstrated that superoxide dismutase (SOD) is required for $T$. vaginalis during the initial phase of oxygen stress [10]. The ironcontaining SOD cannot perform its function normally under iron-deficient situations [11], implying that iron deficiency may induce oxidative stress. In addition to the damaging effects of free radicals, reactive oxygen species (ROS) and reactive nitrogen species (RNS) are also crucial for the signal transduction, that is responsible for the regulation of cellular processes and metabolic activities [12]. Therefore, these molecules might be beneficial for iron-deficient cells. To date, there have been no reports on intrinsic ROS or RNS production or the corresponding signaling pathways involved in iron-deficient T. vaginalis. Hence, we investigated the cellular events regulated by these multi-functional molecules.

Hydrogenosome, a mitochondrial homolog, is the center of energy metabolism as well as the iron-sulfur cluster assembly machinery [13]. Iron-sulfur cluster containing proteins are key molecules responsible for ATP production [14]. In an iron-deficient state, the hydrogenosomes are impaired, affecting the efficiency of energy generation [15]. Additionally, iron deficiency reduces hydrogenosomal membrane potential [16], which is a determinant for the health status of the trichomonad cells [17]. These evidences imply that iron deficiency might cause impairments in cells by disrupting the hydrogenosomal functions. In mammals, redox molecules are associated with the biogenesis and activity of mitochondria [18]. For instance, nitric oxide (NO) regulates the biogenesis of mitochondria via cGMP (cyclic guanosine monophosphate)-dependent signaling [19]. This suggests that redox regulators might also participate in the modulation of hydrogenosomal activity in T. vaginalis.
Until now, the detailed mechanisms modulating the survival of $T$. vaginalis in iron-deficient environments were unclear. In this study, we found that NO dramatically accumulated in iron-deficient $T$. vaginalis. More importantly, we determined that NO production is dependent on the ubiquitin-proteasome system (UPS) and arginine, and that the process maintains the hydrogenosomal membrane potential to enhance the viability of $T$. vaginalis in iron-deficient environments.

\section{Methods}

\section{T. vaginalis culture and treatments}

T. vaginalis ATCC strain 30236 was cultured at $37^{\circ} \mathrm{C}$ in yeast extract, iron-serum (YI-S) medium containing $80 \mu \mathrm{M}$ ferrous ammonium citrate (FAC, Sigma-Aldrich, USA) (iron-rich condition) [20]. Iron-deficient cells were grown in YI-S medium without iron supplementation and treated with $180 \mu \mathrm{M}$ of the iron chelator dipyridyl (DIP, Sigma-Aldrich) at a cell density of $10^{6}$ cells $/ \mathrm{ml}$. The cells for assays were harvested from the mid-log phase of iron-rich cells and the iron-deficient cells were cultured with DIP for $6 \mathrm{~h}$. The trypan blue exclusion assay was used to monitor the growth of cells.

NO synthase inhibitor NG-monomethyl L-arginine (L-NMMA, Sigma-Aldrich, 1 and $3 \mathrm{mM}$ ), proteasome inhibitor MG132 (Sigma-Aldrich, 5 and $10 \mu \mathrm{M}$ ), and arginine (Sigma-Aldrich, $5 \mathrm{mM}$ ) were also added in different experimental groups.

\section{Total RNA extraction}

The total RNA of $T$. vaginalis cultured in iron-rich and -deficient medium was extracted as follows. The cell pellets $\left(2 \times 10^{7}\right.$ cells $)$ were resuspended by adding $1 \mathrm{ml}$ TRI Reagent (Life Technologies) and were incubated at room temperature for $5 \mathrm{~min}$, followed by the addition of $200 \mu \mathrm{l}$ chloroform and incubation at room temperature for 15 min. The RNA fraction was collected after $16,750 \times \mathrm{g}$ centrifugation at $4{ }^{\circ} \mathrm{C}$ for $15 \mathrm{~min}$. Diethylpyrocarbonate (DEPC)-treated $70 \%$ alcohol was used to wash the RNA pellets, and the dried RNA was reconstituted after adding the DEPC-treated water.

\section{Quantitative real-time PCR}

The mRNA was reverse-transcribed to cDNA by reverse transcriptase reactions. The first step contained $5 \mu \mathrm{g}$ total RNA, $50 \mathrm{nM}$ RT primer (oligo-dT, Invitrogen, Life Technologies), and $0.25 \mathrm{mM}$ dNTPs in $10 \mu \mathrm{l}$; the mixture was incubated at $65{ }^{\circ} \mathrm{C}$ for $5 \mathrm{~min}$. cDNA Synthesis Mix $\left(0.75 \mathrm{U} / \mu \mathrm{l}\right.$ ThermoScript ${ }^{\mathrm{Tm}}$ III reverse transcriptase (Invitrogen, Life Technologies), $0.2 \mathrm{U} / \mu \mathrm{l}$ RNase out (Promega, USA), and $0.05 \mathrm{M}$ dithiothreitol (DTT, Sigma-Aldrich) was added to the mixture, and the RNA was converted to cDNA in a series of incubations (25, 50 , and $85{ }^{\circ} \mathrm{C}$ for 5,60 and $15 \mathrm{~min}$, respectively). RNA 
was removed from the RNA-cDNA hybrids after treatment with RNase $\mathrm{H}$ for 20 min. All RNA samples were quantified using a UV Spectrophotometer SMA 1000 (Merinton, China) before experiments.

Real time PCR was performed to validate the expression levels of antioxidants as previously described $[15,21]$ (Additional file 1). Ribosomal protein L8 (TVAG_104490) was used as the internal control for data normalization (forward: TTGCGGTATCAAGATGAACCCAG, reverse: GAACCAAAGCTTTATGCAAGGTTGA). The following reagents composed the reaction mixture: $2 \mathrm{X}$ reaction mix (Ampliqon, Denmark), primers $(0.5 \mu \mathrm{M})$, and $50 \mathrm{ng} \mathrm{cDNA}$ from each condition, with the volume adjusted to $20 \mu \mathrm{l}$ with sterile water. The reaction was performed on an MX3000p (Stratagene, Agilent Technologies), using ROX dye as a reference and SYBR green as a quantifying signal [22]. The data were calculated by MX pro program version 4.10 (Stratagene, Agilent Technologies), representing the relative expression level of each gene in different conditions.

\section{Total antioxidant capacity test}

The total antioxidant capacities of cells cultured in different iron concentrations were determined as described by the manufacturer (BioVision, USA). A total of $2 \times 10^{6}$ cells were collected from each group and lysed by $100 \mu \mathrm{l}$ Radio-ImmunoPrecipitation Assay (RIPA) lysis buffer. After centrifugation at $16,750 \times \mathrm{g}$ for $10 \mathrm{~min}, 15 \mu \mathrm{l}$ of the samples were added and the volume was adjusted to $100 \mu \mathrm{l}$ with the assay diluent. Working reagent $(100 \mu \mathrm{l}$ for each sample) was prepared and then combined with $\mathrm{Cu}^{++}$and assay diluent in a ratio of 1:49. The $100 \mu \mathrm{l}$ of diluted samples and trolox standards $(0,4,8,12,16$, and $20 \mathrm{nmol} /$ well) were mixed with $100 \mu \mathrm{l}$ of working reagent and incubated at room temperature for $30 \mathrm{~min}$. The absorbance at $570 \mathrm{~nm}$ of each sample was measured by using an ELISA reader, SpectraMax M2e (Molecular devices, USA). The values were expressed as $\mathrm{mM}$ of $\mathrm{Cu}^{++}$reduced, converted from standard trolox equivalent.

\section{Reactive oxygen species (ROS) detection}

ROS detection was performed using a previously described approach with slight modification [23]. Briefly, $5 \times 10^{6}$ cells were collected from different cultivations. After wash steps, the cell pellets were treated with $1 \mathrm{ml}$ pre-warmed phosphate buffered saline (PBS), and seeded $100 \mu \mathrm{l}$ cells in the wells of the micro-plate. A final concentration of $1 \mu \mathrm{M}$ chloromethyl-2', 7'dichloroflurescein diacetate (CM-DCFDA, Molecular Probes, Life Technologies) was added into each well, and incubated at $37{ }^{\circ} \mathrm{C}$ for $1 \mathrm{~h}$. Fluorescence signals were detected using an ELISA reader (excitation/ emission $=490 / 525 \mathrm{~nm}$ ).

\section{Intracellular nitric oxide (NO) detection}

NO was detected by following the manufacturer's procedures. A total of $5 \times 10^{6}$ cells were collected from each culture system. After wash steps, cell pellets were resuspended with $1 \mathrm{ml}$ PBS, and the $100 \mu \mathrm{l}$ aliquots of cells were seeded into dark microcentrifuge tubes containing $1 \mu \mathrm{l}$ of $1 \mathrm{mM}$ 4-amino-5methylamino-2', 7'-difluorofluorescein diacetate (DAF-FM DA, Molecular Probes, Life Technologies). After incubation at $37^{\circ} \mathrm{C}$ for $1 \mathrm{~h}$, the loading buffer was washed out, and the cell pellets were resuspended by the addition of $1 \mathrm{ml}$ PBS and then incubated for $30 \mathrm{~min}$. The fluorescent signals were detected with an ELISA reader (excitation/ emission as 495/520 nm) and the fluorescence intensities were recorded.

\section{Next generation sequencing (NGS) and data analysis}

The NGS analysis was performed as previously described [24]. Briefly, the prepared cDNA (iron-rich and -deficient) were fragmented for library construction and sequenced using the Illumina sequencing platform ( $\mathrm{HiSeq}^{\mathrm{Tm}}$ 2000, Illumina, USA). The raw data sets were processed using CLC Genomic workbench (CLC bio) software (version 7). Reads in each gene set were identified with the tool "Mapping to reference" against the G3 reference genome (TrichDB, release-1.3) [25]. All parameters were setup as the recommended defaults. Besides, the length fractions and similarity were changed to 0.9 and 0.8 , respectively. Reads Per Kilobase per Million mapped reads (RPKM) of each gene were calculated and used as the normalized gene expression level (Additional file 2). The basic information generated from this analysis is shown in Additional file 3.

\section{Proteasome activity assay}

Proteasome activities of different iron availabilities were determined by a fluorescent approach (proteasome activity fluorometric assay kit, BioVision) following the manufacturer's protocol. The iron-rich and -deficient cultured cells $\left(2 \times 10^{6}\right)$ were lysed in $100 \mu \mathrm{l}$ of $0.5 \%$ NP-40 (Sigma-Aldrich). The reaction mixture contained $10 \mu \mathrm{l}$ of testing samples, $90 \mu \mathrm{l}$ assay buffer, and $1 \mu \mathrm{l}$ of proteasome substrate in the wells of the micro-plate. MG132 (proteasome inhibitor) treated group was used as the negative control for calibration. The fluorescence signal can be detected when the substrate is digested by proteasome in cell lysates. After 10 and $30 \mathrm{~min}$ of incubation at $37{ }^{\circ} \mathrm{C}$, the values of experimental groups as well as the aminomethylcoumarin (AMC) standards $(0,20,40,60,80$, and $100 \mathrm{pmol} /$ well) were measured simultaneously by using an ELISA reader (excitation/emission $=350 / 440 \mathrm{~nm}$ ). The kinetics of the proteasome activity was generated after subtracting fluorescence intensities detected from 2 time points of each sample. The values were expressed in $\mathrm{nmol} / \mathrm{min} / \mathrm{ml}$. 


\section{Hydrogenosomal membrane potential measurement}

The hydrogenosomal membrane potential was measured as previously described [26]. We harvested $1 \times 10^{6}$ cells from each of the treatments (DIP only, DIP plus MG132, DIP plus MG132 and arginine) after $6 \mathrm{~h}$ of drug exposure. The cell pellets were resuspended in $1 \mathrm{ml}$ PBS. No stained cell (blank) without JC-1 (Molecular Probes, Life Technologies) treatment was prepared for flow cytometry adjustment. All testing cells included a negative control (treated with mitochondrial membrane potential disrupter carbonyl cyanide 3-chlorophenyl hydrazone (CCCP) in $50 \mu \mathrm{M})$ were treated with $2 \mu \mathrm{M}$ JC1 , incubating at $37{ }^{\circ} \mathrm{C}$ for $30 \mathrm{~min}$. The cells were washed once with PBS, and the cell was then resuspended with 0.5 $\mathrm{ml}$ PBS. The red fluorescence intensities alternations in experimental groups were detected by flow cytometry analysis.

\section{Statistical analysis}

Student's t-tests were performed on the quantified data derived from biological assays, using GraphPad Prism 5 software. Asterisks were used to represent the significance of each assay as determined through the $\mathrm{p}$ value $\left(*: p<0.05 ;{ }^{* * *}: p<0.01 ;{ }^{* * * *}: p<0.001\right)$.

\section{Results}

\section{$T$. vaginalis survives in iron-deficient condition}

It is known that iron is essential for cell proliferation in T. vaginalis. Iron restriction causes an increase in doubling time and a decrease in the maximum cell density [27]. Until now, no report indicates the viability of $T$. vaginalis upon iron deficiency. To monitor the cell viability under iron-deficient condition, we cultured the cells with a higher initial cell density $\left(10^{6}\right.$ cells $\left./ \mathrm{ml}\right)$ in the medium containing $180 \mu \mathrm{M}$ DIP $[24,28]$. The iron-rich cells reached the maximum cell density of approximately $3.5 \times 10^{6}$ (cells $\left./ \mathrm{ml}\right)$ at $6 \mathrm{~h}$ after inoculation, followed by a rapid decline (Fig. 1a). The viable cells were reduced to $1 \times 10^{5}$ (cells $/ \mathrm{ml}$ ) at approximately $50 \mathrm{~h}$ in iron-rich cultivation. In contrast, we found that the maximum cell density of iron-deficient cells was 2-fold $\left(\sim 1.5 \times 10^{6}\right.$ cells $\left./ \mathrm{ml}\right)$ less than that of iron-rich cells (Fig. 1b). Interestingly, our result showed that the survival of iron-deficient cells was further extended to $66 \mathrm{~h}$ with a cell density of $1 \times 10^{5}$ viable cells per $\mathrm{ml}$. This phenomenon reflects that although iron deficiency affects cell proliferation, $T$. vaginalis is capable of adapting to an iron-deficient environment and survives for a longer period. However, the underlying mechanism responsible for cell survival during iron deficiency is still unclear, which might be an important issue in elucidating how $T$. vaginalis establishes and maintains infection in the vaginal region.

\section{NO is accumulated in iron-deficient $T$. vaginalis}

Previous studies have indicated that iron-deficient cells exhibit up- and down-regulation of thiol- and irondependent antioxidant defense systems, respectively $[15,21]$. We confirmed the expression patterns of the irondependent antioxidants SOD (TVAG_039980, TVAG_12 0340) and rubrerythrin (TVAG_064490, TVAG_275660) and the thiol-dependent thioredoxin peroxidase (TVAG_ 114310, TVAG_455310) in cells cultured under ironrich and -deficient conditions by using quantitative RT-PCR. The results revealed a trend similar to previous studies (Additional file 4). Furthermore, we examined the cellular reducing power in iron-rich and-deficient cells by measuring the amount of copper reduction $\left(\mathrm{Cu}^{++}\right.$to $\left.\mathrm{Cu}^{+}\right)$, which is used as a general indicator for antioxidant capacity. As shown in Fig. 2a, there is a
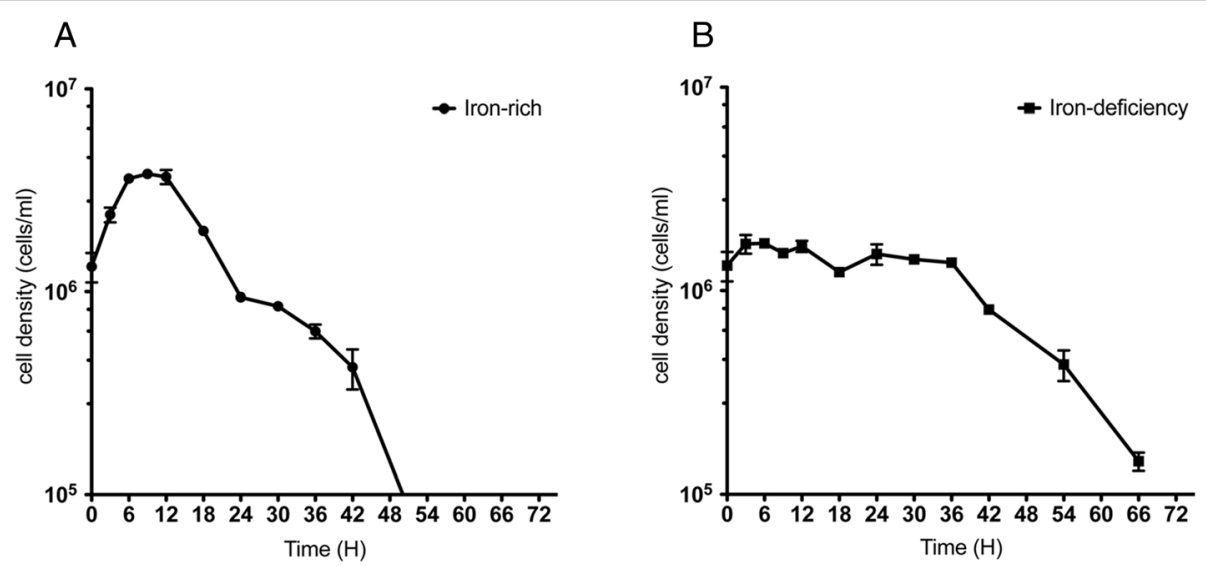

Fig. 1 T. vaginalis extends the survival time when cultured under iron-deficient conditions. The growth of cells cultured in iron-rich (IR, $80 \mu M$ FAC, a) and -deficient (ID, $180 \mu \mathrm{M}$ DIP, b) media. The number of viable cells was monitored every three hours using the trypan blue exclusion assay. The initial cell density is $1 \times 10^{6} \mathrm{cells} / \mathrm{ml}$. The data are presented as the mean \pm SD of three independent experiments 

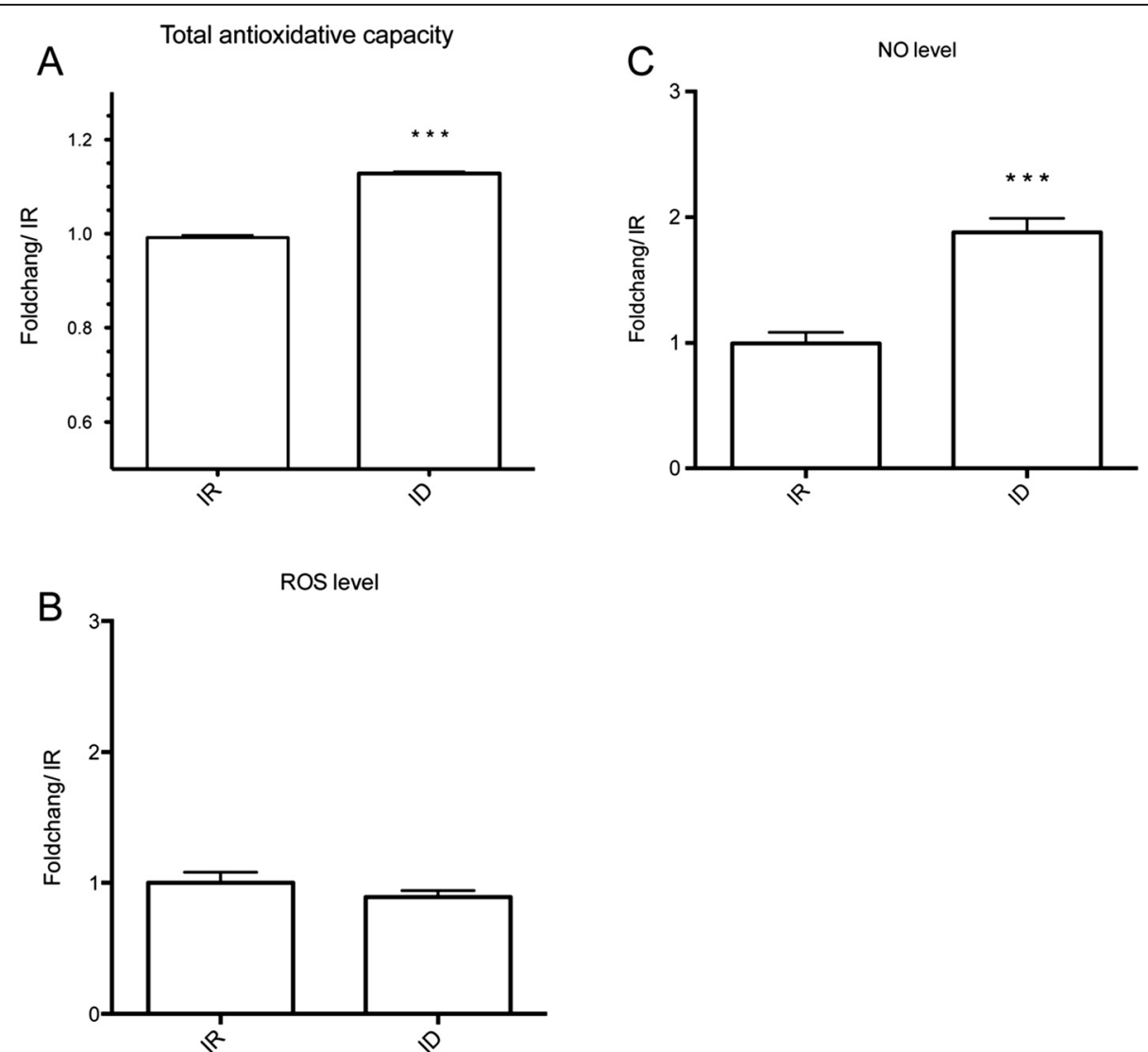

Fig. 2 NO accumulated in iron-deficient T. vaginalis. a Antioxidant capacity of iron-rich (IR) and -deficient (ID) cells representing the reducing power $\left(\mathrm{Cu}^{++}\right.$to $\left.\mathrm{Cu}^{+}\right)$of each lysate. ROS (CM-DCF DA) b and NO (CM-DAF FM) $\mathbf{c}$ were examined. IR, iron rich $(80 \mu M$ FAC); ID, iron deficient $\left(180 \mu \mathrm{M}\right.$ DIP). The data are presented as the mean \pm SD of three independent experiments. ${ }^{* * *} p<0.001$, compared with the IR group

significant increase in copper reduction in the irondeficient group compared with iron-rich group. Together with quantitative RT-PCR analysis of multiple antioxidant genes, these data confirm that the antioxidative response of iron-deficient cells is stronger than that of iron-rich cells.

To understand whether the redox molecules increased simultaneously with antioxidants upon iron deficiency, we used individual fluorescence indicators to detect ROS and RNS (NO). As shown in Fig. 2b, there is no significant difference in ROS production between iron-rich and -deficient groups. Surprisingly, the amount of NO is elevated when the environmental iron concentration decreased (Fig. 2c), with an approximate 2-fold increase in iron-deficient conditions. These data suggest that the increase of intracellular NO production and the antioxidants for redox maintenance in $T$. vaginalis occur under iron-deficient conditions. The accumulated $\mathrm{NO}$ and the corresponding signaling pathways were likely crucial for iron-deficient cells; however, limited information is known about the relationship of $\mathrm{NO}$ and iron deficiency in T. vaginalis.
Transcriptomics analysis revealed NO-related responses in iron-restricted $T$. vaginalis

Previous studies utilizing NGS-based transcriptomics analysis in $T$. vaginalis provided insights into the detailed mechanical information [10, 24]. Therefore, this technique creates the opportunity to identify the novel genes or pathways in T. vaginalis associated with iron deficiency. To date, little is known about the biological functions of intrinsic NO in T. vaginalis, and the massive gene families make it difficult to discern the participating genes. We performed an NGS-based transcriptomics analysis of the iron-deficient cells compared with iron-rich cells to monitor the NO-related events. A total of 51,519,438 and $53,444,250$ high quality reads (100 bases in length) were generated from the iron-rich and -deficient cDNA libraries, respectively (Additional file 3). These data sets covered approximately 100 -fold of the $T$. vaginalis genome that contains more than 60,000 genes. After mapping the reads to the $T$. vaginalis genome, there were 28,256 and 33,976 protein-coding genes expressed in iron-rich and -deficient cells, respectively (Additional file 3). Table 1 showed the most significantly up-regulated genes in iron-deficient 
Table 1 The most expressed genes in iron-deficient T. vaginalis

\begin{tabular}{|c|c|c|c|}
\hline GenelD & TrichDB Annotation & RPKM (IR) & RPKM (ID) \\
\hline \multicolumn{4}{|c|}{ METABOLIC ENZYMES } \\
\hline TVAG_171090 & Malate dehydrogenase, putative & 1171.96 & $19,482.82$ \\
\hline TVAG_344880 & Alcohol dehydrogenase, putative & 115.8 & $3,902.75$ \\
\hline TVAG_038440 & Fructose-bisphosphate aldolase, putative & 63.12 & $1,715.45$ \\
\hline TVAG_196700 & Glutamate dehydrogenase, putative & 41 & $1,787.34$ \\
\hline \multicolumn{4}{|c|}{ OXIDOREDUCTASES } \\
\hline TVAG_167830 & Regulator of cell morphogenesis and NO signaling (RCMNS) (metal ion binding) & 237.68 & $2,776.24$ \\
\hline TVAG_107080 & 4-carboxymuconolactone decarboxylase 2 & 11.21 & $3,030.84$ \\
\hline TVAG_256720 & 4-carboxymuconolactone decarboxylase 1 & 2.82 & $3,363.03$ \\
\hline TVAG_336320 & Hydroxylamine reductase, putative & 70.86 & 1089.89 \\
\hline \multicolumn{4}{|c|}{ PROTEOLYTIC PROCESSES } \\
\hline TVAG_184150 & Ubiquitin, putative & 113.42 & 1563.65 \\
\hline TVAG_476160 & Clan MG, family M24, aminopeptidase P-like metallopeptidase & 77.07 & 1534.83 \\
\hline TVAG_386080 & Clan MG, family M24, aminopeptidase P-like metallopeptidase & 129.25 & 7169.21 \\
\hline \multicolumn{4}{|l|}{ OTHERS } \\
\hline TVAG_469020 & Biotin synthase, putative & 4.15 & $1,508.21$ \\
\hline TVAG_321740 & Conserved hypothetical protein & 67.62 & 8933.47 \\
\hline TVAG_059980 & Conserved hypothetical protein & 102.07 & $1,355.29$ \\
\hline TVAG_307440 & Conserved hypothetical protein & 7.34 & $1,956.57$ \\
\hline TVAG_491130 & Conserved hypothetical protein & 75.06 & $2,060.76$ \\
\hline TVAG_488900 & Conserved hypothetical protein & 1.48 & $2,220.44$ \\
\hline
\end{tabular}

The genes listed in this table are only highly expressed (RPKM > 1000, fold-change >10) in iron-deficient (ID) cells compared to iron-rich (IR) cells. RPKM, reads per kilobase per million mapped reads

cells (RPKM $>1000$ and 10-fold higher than that in iron-rich cells), including metabolic enzymes, proteolysis, oxidoreductases, and hypothetical genes. Among the highly expressed genes, hydroxylamine reductase (TVAG_336320), carboxymuconolactone decarboxylases (TVAG_107080, 256720), and regulators of cell morphogenesis and NO signaling (TVAG_167830) are all related to NO signaling pathways in other organisms [29-31]. The expression patterns of these genes were also validated by quantitative RT-PCR (Additional file 5). This further prompted us to study the roles of NO in iron-deficient $T$. vaginalis.

\section{NO production is via arginine- and proteasome-dependent pathway}

It has been suggested that $T$. vaginalis probably uses arginine deiminase (ADI), with no putative nitric oxide synthase encoded, to produce NO when the culture is supplemented with arginine in aerobic conditions [32]. According to our transcriptomics data, ADIs (TVAG_344520, TVAG_467820, and TVAG_183850) exhibited up-regulation in iron-deficient conditions (Additional file 2). To understand whether arginine is the substrate for $\mathrm{NO}$ production, we treated the iron-deficient cells with 1 and $3 \mathrm{mM}$ arginine derivative L-NG-monomethyl arginine (L-NMMA) and monitored the NO levels. As shown in Fig. 3a, the NO level in iron-deficient cells treated with L-NMMA decreased in a dose-dependent manner after $3 \mathrm{~h}$ of treatment, with an approximately $20 \%$ reduction in NO in cells treated with 3 mM L-NMMA compared with the untreated group, suggesting the importance of arginine for $\mathrm{NO}$ production in iron-deficient $T$. vaginalis. The effect of L-NMMA on NO reduction could not be sustained for a long period in iron-deficient cells (Additional file 6), suggesting that either more arginine is catalyzed for NO production or L-NMMA is consumed at later time points.

The ubiquitin-proteasome system (UPS) is thought to be the machinery for maintaining an intracellular arginine pool for NO production [33]. Hence, we investigated whether the UPS plays a key role in NO production in $T$. vaginalis during iron deficiency. Indeed, the proteasome activity is significantly increased in iron-deficient cells (Fig. 3b). To elucidate the correlation between proteolysis and NO production, we detected the NO level in iron-deficient cells treated with or without the proteasome inhibitor, MG132. The data revealed that the cells treated with MG132 have a decrease of $20 \%$ in NO 


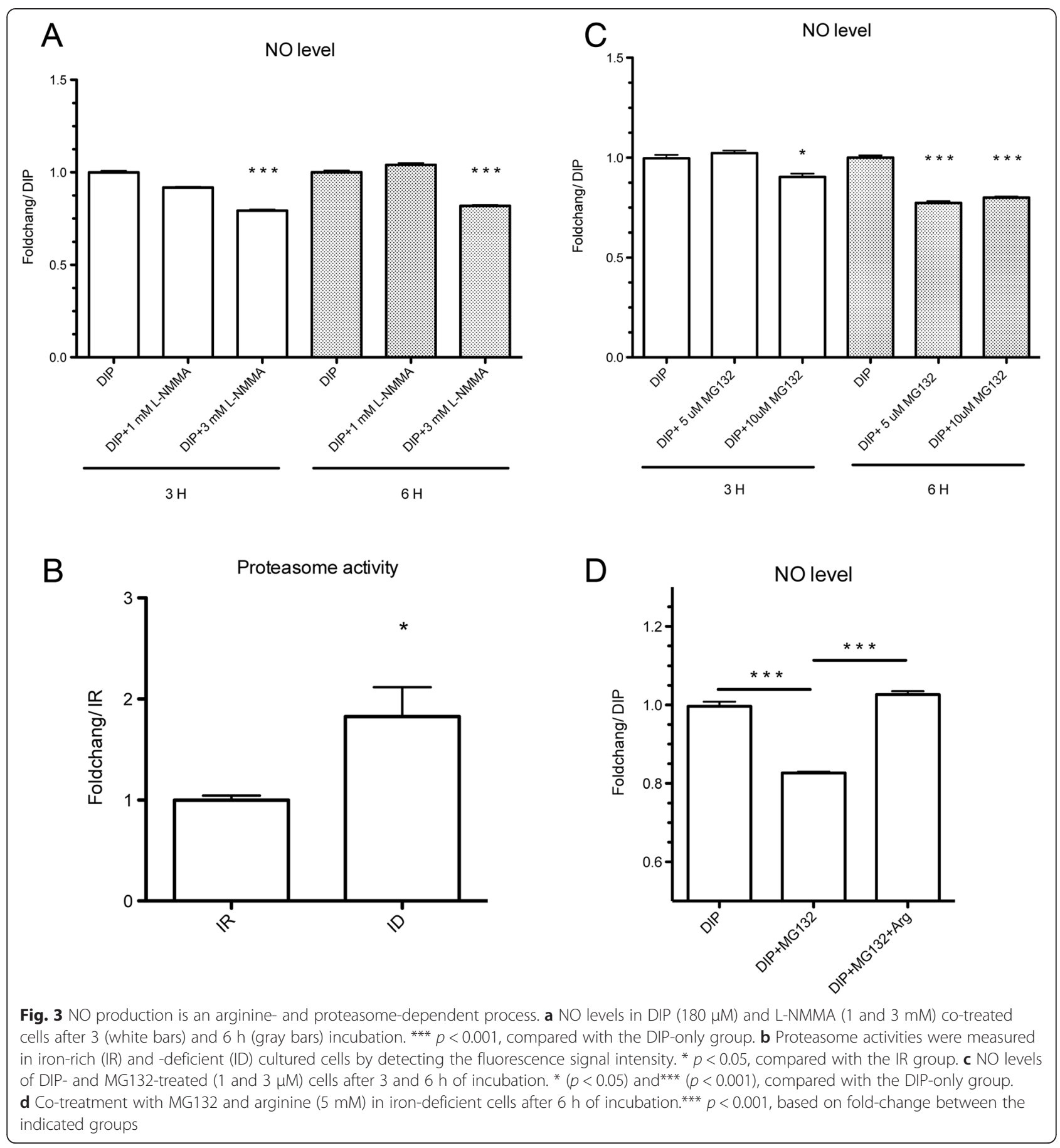

production in a time- and dose-dependent manner (Fig. 3c). NO reduction in the MG132-treated group can be restored by the addition of arginine (Fig. 3d), confirming the function of UPS in arginine pool maintenance and $\mathrm{NO}$ production in iron-deficient $T$. vaginalis.

Because the change in the amount of NO in MG132treated cells can be totally reversed by arginine, and there is a longer inhibitory effect of MG132 on NO production compared with L-NMMA treated cells
(Additional file 6), we thereby used MG132 to deplete NO production and clarify the roles of $\mathrm{NO}$ in iron-deficient cells. Taken together, we demonstrated that NO production depends on arginine and the UPS activity in T. vaginalis under iron-deficient conditions.

\section{NO maintains cell survival in iron-deficient $T$. vaginalis}

To verify the role of $\mathrm{NO}$ in the survival of $T$. vaginalis under iron-deficient conditions, we monitored the growth 
of cells treated with or without MG132 by the trypan blue exclusion assay. As shown in Fig. 4, the viability of irondeficient cells can be maintained at least $24 \mathrm{~h}$ after DIP treatment with a cell density of $\sim 1 \times 10^{6}$ (cells $/ \mathrm{ml}$ ), whereas combined treatment with MG132 and DIP reduced the viable cells to $\sim 3 \times 10^{5}$ (cells $\left./ \mathrm{ml}\right)$ at $21 \mathrm{~h}$. Interestingly, the number of viable cells recovered by approximately 2 -fold at $21 \mathrm{~h}$ when the iron-deficient cells were co-treated with MG132 and arginine compared with MG132 treatment alone, suggesting that UPS-generated NO is important for cell survival. The UPS-inhibited cells could be rescued by the addition of single amino acid arginine, emphasizing the crucial function of UPS in NO production in iron-deficient $T$. vaginalis. In other words, this result demonstrates that $\mathrm{NO}$ is a pivotal molecule for cell survival when $T$. vaginalis resides in iron-deficient environments.

\section{NO enhances hydrogenosomal membrane potential in $T$. vaginalis upon iron deficiency}

Previous studies have proven that NO activates cGMP signaling to modulate mitochondrial biogenesis and activity in mammals [34]. Mitochondrial membrane potential is believed to be a determinant of metabolic activity and health status of a cell [35]. We utilized a cellpermeable dye JC-1 to monitor the hydrogenosomal membrane potential of $T$. vaginalis, which reflects the hydrogenosomal functions $[17,26]$. Using JC-1 staining, we monitored the fluctuations in the red fluorescencecontaining cells, which possessed hydrogenosomes with

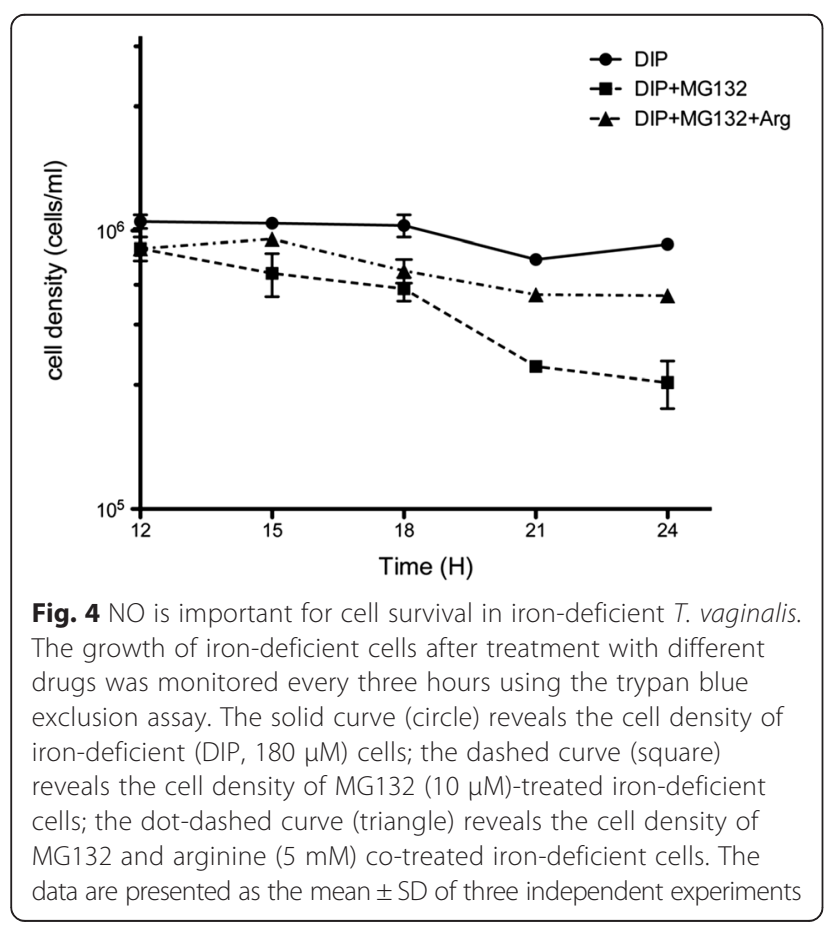

high membrane potential, in different iron concentrations. We used the cells treated with CCCP, a disruptor of mitochondrial membrane potential, as a negative control. As shown in Fig. 5a and b, the fluorescence intensities were reduced in the CCCP-treated cells, indicating that the measurement is suitable for analysis of hydrogenosomal membrane potential. We found that the red fluorescence intensity in MG132 treated iron-deficient cells was decreased significantly $(p<0.01)$ compared with untreated cells (Fig. 5b). Interestingly, the MG132mediated reduction in red signal can be recovered by the addition of arginine (Fig. 5b), suggesting that NO maintains the hydrogenosomal membrane potential in iron-deficient $T$. vaginalis. These observations indicate that the hydrogenosomal membrane potential of MG132treated cells can be reversed after co-treatment with arginine, confirming that $\mathrm{NO}$ functions in hydrogenosomal membrane potential maintenance. These data demonstrate that NO serves as a "keeper" for maintaining the functions of the hydrogenosome, which is positively correlated with prolonged survival in iron-deficient $T$. vaginalis.

\section{Discussion}

T. vaginalis has been shown to adapt to environmental changes in the vaginal region, such as metabolic reprogramming and autophagy in response to glucose restriction [24]. Similarly, the shift of the energy production pathway from hydrogenosome to glycolysis in irondeficient conditions has been demonstrated $[15,36]$ (Table 1). In the present work, we found that T. vaginalis can survive for an extended time in the environment without sufficient iron supplementation. Nevertheless, the mechanisms involved in the adaptation in irondeficient situations are still largely unknown.

The up-regulation of NO in parallel with elevated antioxidant capacity suggests that $\mathrm{NO}$ signaling might be critical for iron-deficient $T$. vaginalis [37]. NO serves as a regulator to protect the cells from iron shortagemediated damages [38]. For instance, accumulated NO functions in adjusting iron utilization and preventing oxidative stresses in iron-restricted plants [39, 40]. NO accelerates the release of iron from ferritin to re-balance the free iron concentration in iron deficient anemia models [41].

NO production from arginine-dependent pathway has been suggested in a previous study [32]. Nevertheless, it is still unknown how NO produced in T. vaginalis. In our work, we determined that $T$. vaginalis used arginine as the substrate for NO production under iron-deficient conditions without additional arginine supplementation. UPS, proteolytic machinery that commonly exists in eukaryotic and prokaryotic cells, functions in protein turnover and quality control [42]. The conserved ubiquitin-conjugating system and proteasome subunits 

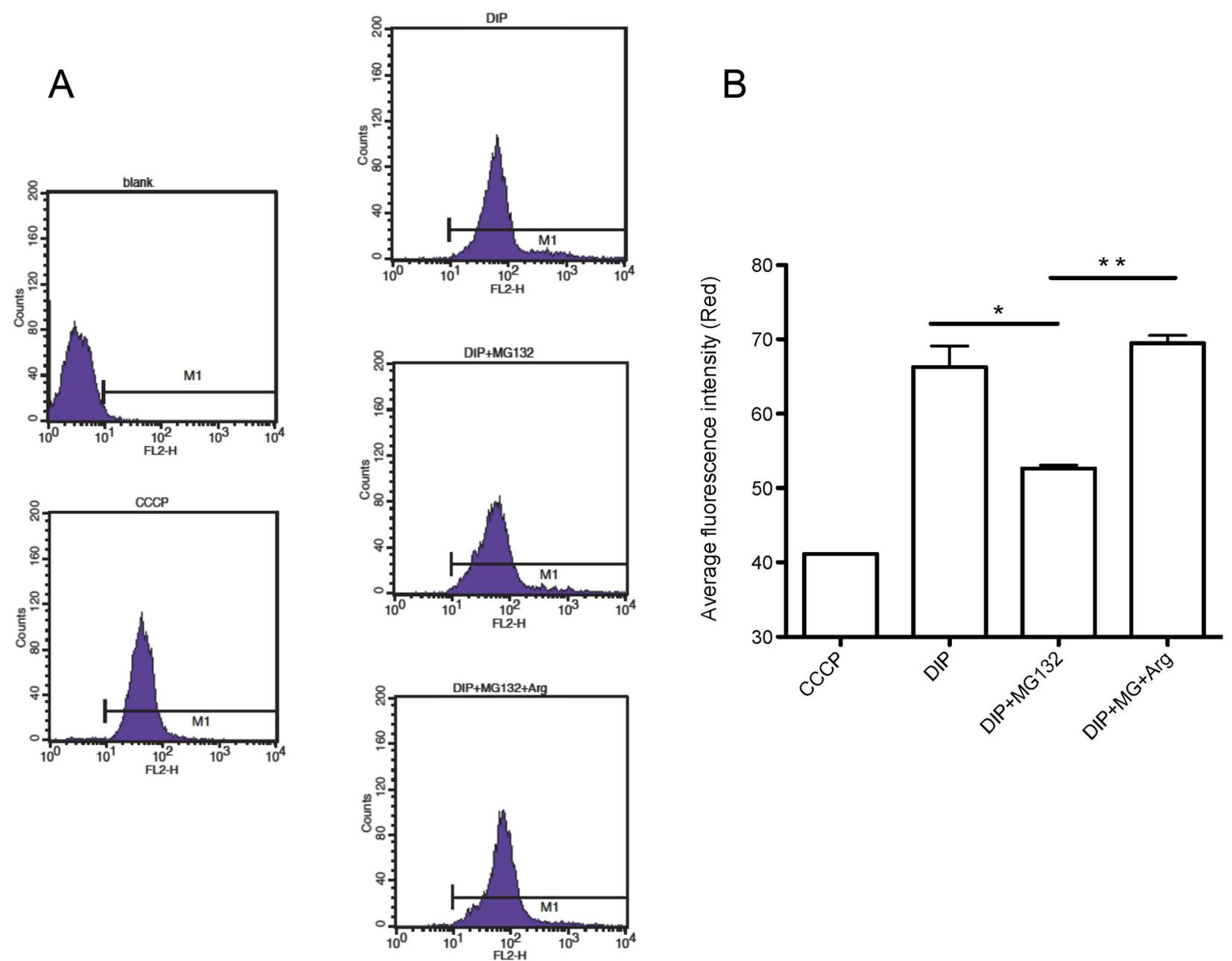

Fig. 5 NO maintains the hydrogenosomal membrane potential of iron-deficient T. vaginalis. a The histograms indicate the red signal of the cells treated with DIP $(180 \mu \mathrm{M})$, DIP-MG132 $(10 \mu \mathrm{M})$, and DIP-MG132-arginine $(5 \mathrm{mM})$. Red fluorescence represents a hydrogenosome with high membrane potential. The red fluorescence intensities of negative control group (CCCP treated cells, $50 \mu \mathrm{M})$ and experimental groups were detected by using flow cytometry after JC-1 staining. b Quantification data of the red fluorescence intensities (geometric mean) in panel a are shown. All tested groups were collected $6 \mathrm{~h}$ after drug treatments, and the data are presented as the mean \pm SD of three independent experiments. $*(p<0.05)$ and ${ }^{* *}(p<0.01)$, based on the differences between the indicated groups

can be found in the genome of $T$. vaginalis. However, none of them have been characterized. The UPS generates amino acid for cellular functions that have been examined in mammals, such as cysteine and arginine $[33,43]$. We illustrated that $T$. vaginalis utilizes UPSderived arginine for NO production under iron-deficient conditions. We also found that the UPS plays a pivotal role in the survival of iron-deficient cells, which correlated with NO production. This finding suggests that the functional role of UPS in NO production could be conserved in $T$. vaginalis.

Previous study suggests that $T$. vaginalis can also generate energy from arginine provided by either UPS or exogenous arginine via the arginine dihydrolase pathway [44]. However, according to our transcriptomics analysis, the compensatory responses were raised for energy production due to compromised hydrogenosomal energy metabolism of iron-deficient cells, such as carbohydrate metabolism (Table 1). In addition, the arginine dihydrolase pathway only contributes $10 \%$ of ATP that generated from glycolysis [44]. Thus, we believe that exogenous arginine may trigger $\mathrm{NO}$-related responses rather than ATP production to protect $T$. vaginalis from iron deficiency.

$\mathrm{NO}$ is a second messenger that controls cellular functions via triggering the production of cGMP [45]. The cGMP-regulated pathways are likely the important downstream effect of accumulated NO. We have monitored the cGMP levels in iron-deficient cells, which are $10 \%$ greater than iron-rich cells (data not shown). The 
increased cGMP directly activates cGMP-dependent protein kinase (known as protein kinase G or PKG), which participates in signaling related to cell survival [46]. Among the 144 members of the protein kinase AGC family in the genome of $T$. vaginalis, no specific PKG was annotated [25]. It is difficult to pinpoint which gene is responsible for the cGMP-dependent reactions because there is high sequence similarity within the catalytic domain. Additionally, PKG is the potential drug target for Toxoplasma and Plasmodium spp. treatment $[47,48]$. Therefore, the identification and characterization of PKG related to NO metabolism in T. vaginalis, especially under iron deficiency, is a crucial task in the future.

The NO-dependent increase in cGMP also regulates mitochondrial biogenesis in mammals [19, 45]. Previous studies demonstrated that iron-deficiency leads to a reduction in hydrogenosomal activity [16]. Here, we found that if $\mathrm{NO}$ production in iron-deficient cells is reduced, the membrane potential of hydrogenosome is further decreased. This suggests that NO is crucial for the functional maintenance of iron-restricted hydrogenosome. The mitochondrial membrane potential is a determinant for cell health [35]. Likewise, the linkage between hydrogenosomal membrane potential and cell death in $T$. vaginalis has been determined [17]. In fact, low mitochondrial membrane potential represents increased permeability, which is correlated with apoptosis in mammalian cells [35]. Accordingly, we proposed that NO-enhanced hydrogenosomal membrane potential is critical for cell survival under irondeficiency.

\section{Conclusions}

In conclusion, we demonstrate, for the first time, that T. vaginalis utilized a NO-dependent regulatory network to survive in iron-deficient situations (Fig. 6). Once T. vaginalis encounters iron-limited environments, the protist generates more NO via proteasome-dependent pathway. The UPS-derived arginine is the substrate for NO production [33]. The generation of NO possibly takes place in the hydrogenosome since ADI, the

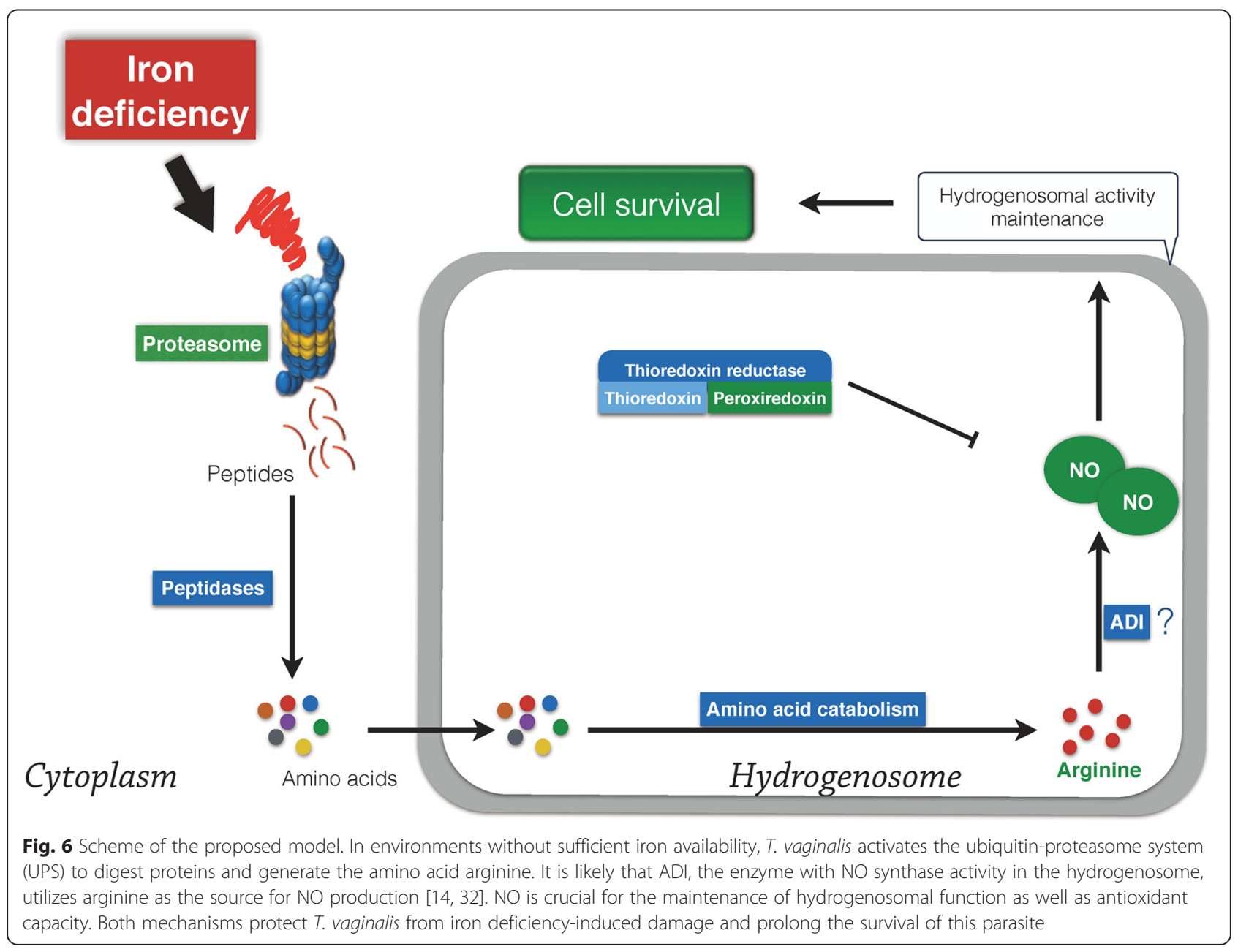


enzyme with NO synthase activity, is found in this organelle [14, 32]. NO is a pivotal factor that modulates the hydrogenosomal membrane potential to protect cells from death. These mechanisms are vital for $T$. vaginalis to adapt to the continuous alternation of iron in the vaginal region, which is beneficial for establishment of an infection and parasitization.

\section{Additional files}

\section{Additional file 1: Primer sets for quantitative RT-PCR. \\ Additional file 2 Transcriptomic analysis of $T$. vaginalis under different iron concentrations by RNA-seq. \\ Additional file 3 Summary of NGS analysis.}

Additional file 4: Iron-dependent expression of antioxidants in $T$. vaginalis. The expression levels of antioxidative defense systems in cells cultured under different iron concentrations were determined by using quantitative RT-PCR. IR, iron rich ( $80 \mu \mathrm{M} F A C)$; ID, iron deficiency (180 M DIP). SOD, superoxide dismutase; Rbr, rubrerythrin; TrxP, thioredoxin peroxidase.

Additional file 5: Validation of NO-related genes identified from transcriptomics analysis. The expression levels of iron deficiencyinduced genes were verified by using quantitative RT-PCR. IR, iron rich

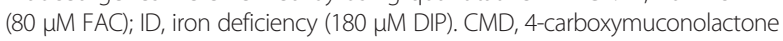
decarboxylase; HCP, hybrid cluster protein or hydroxylamine reductase; RCMNS, regulator of cell morphogenesis and NO signaling.

Additional file 6: $\mathrm{NO}$ levels in iron-deficient $T$. vaginalis treated with NOS and proteasome inhibitors. NO levels in iron-deficient cells treated with the NOS inhibitor (L-NMMA, $3 \mathrm{mM}$ ) and the proteasome inhibitor (MG132, $10 \mu \mathrm{M})$ for $12 \mathrm{~h}$.

\section{Abbreviations}

ROS: Reactive oxygen species; RNS: Reactive nitrogen species; NO: Nitric oxide; CGMP: cyclic guanosine monophosphate; L-NMMA: L-NG-monomethyl arginine; SOD: Superoxide dismutase; UPS: Ubiquitin-proteasome system; YI-S medium: Yeast extract, iron-serum medium; DIP: Dipyridyl; DEPC: Diethylpyrocarbonate; dT: deoxythymidine; dNTP: deoxynucleotide; CDNA: complementary DNA; RNase: Ribonuclease; DTT: Dithiothreitol; RTPCR: Real-time polymerase chain reaction; PBS: Phosphate buffered saline; CM-DCF DA: Chloromethyl-2', 7'-dichloroflurescein diacetate; ELISA: Enzymelinked immunosorbent assay; DAF-FM DA: 4-amino-5- methylamino-2', 7'difluorofluorescein diacetate; NGS: Next generation sequencing; RPKM: Reads per kilobase per million mapped reads; AMC: Aminomethylcoumarin; CCCP: Carbonyl cyanide 3-chlorophenyl hydrazine; Rbr: Rubrerythrin; TrxP: Thioredoxin peroxidase; ADI: Arginine deiminase.

\section{Competing interests}

The authors declare that they have no competing interests.

\section{Authors' contributions}

WHC and KYH designed the experiments; PJH and YKF analyzed the NGS data; WHC and JHH performed the experiments; WHC and KYH wrote the manuscript; $\mathrm{CHC}$ and PT revised the manuscript. All the authors read and approved the final version of the manuscript.

\section{Acknowledgements}

We thank Dr. Po-Liang Lai and Ms. Chia-Jung Wu for help in flow cytometry analysis. The present study was supported by the Chang Gung Memorial Hospital Research Funding program (CMRPD3D0181) and from Ministry of Science and Technology, Taiwan (104-2320-182-025-MY3).

\section{Author details}

'Graduate Institute of Biomedical Sciences, College of Medicine, Chang Gung University, Kweishan, Taoyuan, Taiwan. ${ }^{2}$ Molecular Regulation and Bioinformatics Laboratory, Department of Parasitology, College of Medicine, Chang Gung University, Kweishan, Taoyuan, Taiwan. ${ }^{3}$ Molecular Medicine
Research Center, Chang Gung University, Kweishan, Taoyuan, Taiwan. ${ }^{4}$ Bioinformatics Center, Chang Gung University, Kweishan, Taoyuan, Taiwan. ${ }^{5}$ Molecular Infectious Disease Research Center, Chang Gung Memorial Hospital, Kweishan, Taoyuan, Taiwan.

Received: 16 February 2015 Accepted: 9 July 2015

Published online: 25 July 2015

\section{References}

1. Schwebke JR, Burgess D. Trichomoniasis. Clin Microbiol Rev. 2004;17:794-803.

2. Alderete JF, Provenzano D, Lehker MW. Iron mediates Trichomonas vaginalis resistance to complement lysis. Microb Pathog. 1995;19:93-103.

3. Alvarez-Sánchez ME, Solano-González E, Yañez-Gómez C, Arroyo R. Negative iron regulation of the CP65 cysteine proteinase cytotoxicity in Trichomonas vaginalis. Microbes Infect. 2007:9:1597-605.

4. Crouch ML, Benchimol M, Alderete JF. Binding of fibronectin by Trichomonas vaginalis is influenced by iron and calcium. Microb Pathog. 2001;31:131-44

5. Sehgal R, Goyal K, Sehgal A. Trichomoniasis and lactoferrin: future prospects. Infect Dis Obstet Gynecol. 2012;2012:1-8.

6. Figueroa-Angulo EE, Rendón-Gandarilla FJ, Puente-Rivera J, Calla-Choque JS, Cárdenas-Guerra RE, Ortega-López J, et al. The effects of environmental factors on the virulence of Trichomonas vaginalis. Microb Infect. 2012;14:1411-7.

7. Heath JL, Weiss JM, Lavau CP, Wechsler DS. Iron deprivation in cancerpotential therapeutic implications. Nutrients. 2013;5:2836-59.

8. Vale-Costa S, Gomes-Pereira S, Teixeira CM, Rosa G, Rodrigues PN, Tomás A, et al. Iron overload favors the elimination of Leishmania infantum from mouse tissues through interaction with reactive oxygen and nitrogen species. PLoS Negl Trop Dis. 2013;7, e2061.

9. Storr SJ, Woolston CM, Zhang Y, Martin SG. Redox environment, free radical, and oxidative DNA damage. Antioxid Redox Sign. 2013;18:2399-408.

10. Gould SB, Woehle C, Kusdian G, Landan G, Tachezy J, Zimorski V, et al. Deep sequencing of Trichomonas vaginalis during the early infection of vaginal epithelial cells and amoeboid transition. Int J Parasitol. 2013;43:707-19.

11. Chakraborty S, Tewari S, Sharma RK, Narula SC, Ghalaut PS, Ghalaut V. Impact of iron deficiency anemia on chronic periodontitis and superoxide dismutase activity: a cross-sectional study. J Periodontal Implant Sci. 2014:44:57-64

12. Bashan N, Kovsan J, Kachko I, Ovadia H, Rudich A. Positive and negative regulation of insulin signaling by reactive oxygen and nitrogen species. Physiol Rev. 2009;89:27-71.

13. Makiuchi T, Nozaki T. Highly divergent mitochondrion-related organelles in anaerobic parasitic protozoa. Biochimie. 2013;100:1-15.

14. Schneider RE, Brown MT, Shiflett AM, Dyall SD, Hayes RD, Xie Y, et al. The Trichomonas vaginalis hydrogenosome proteome is highly reduced relative to mitochondria, yet complex compared with mitosomes. Int J Parasitol. 2011:41:1421-34

15. Beltrán NC, Horváthová L, Jedelský PL, Šedinová M, Rada P, Marcinčiková M, et al. Iron-induced changes in the proteome of Trichomonas vaginalis hydrogenosomes. PLoS One. 2013;8, e65148.

16. Kim Y-S, Song H-O, Choi I-H, Park S-J, Ryu J-S. Hydrogenosomal activity of Trichomonas vaginalis cultivated under different iron conditions. Korean J Parasitol. 2006:44:373-8.

17. Chose O, Noël C, Gerbod D, Brenner C, Viscogliosi E, Roseto A. A form of cell death with some features resembling apoptosis in the amitochondrial unicellular organism Trichomonas vaginalis. Exp Cell Res. 2002;276:32-9.

18. Yoboue ED, Devin A. Reactive oxygen species-mediated control of mitochondrial biogenesis. Int J Cell Biol. 2012;2012:403870.

19. Nisoli E, Clementi E, Paolucci C, Cozzi V, Tonello C, Sciorati C, et al. Mitochondrial biogenesis in mammals: the role of endogenous nitric oxide. Science. 2003;299:896-9.

20. Diamond LS, Clark CG, Cunnick CC. YI-S, a casein-free medium for axenic cultivation of Entamoeba histolytica, related Entamoeba, Giardia intestinalis and Trichomonas vaginalis. J Eukaryot Microbiol. 1995;42:277-8.

21. Horváthová L, Safarikova L, Basler M, Hrdý I, Campo NB, Shin J-W, et al. Transcriptomic identification of iron-regulated and iron-independent gene copies within the heavily duplicated Trichomonas vaginalis genome. Genome Biol Evol. 2012;4:905-17. 
22. Huang K-Y, Huang P-J, Ku F-M, Lin R, Alderete JF, Tang P. Comparative transcriptomic and proteomic analyses of Trichomonas vaginalis following adherence to fibronectin. Infect Immun. 2012;80:3900-11.

23. Husain A, Sato D, Jeelani G, Soga T, Nozaki T. Dramatic increase in glycerol biosynthesis upon oxidative stress in the anaerobic protozoan parasite Entamoeba histolytica. PLoS Negl Trop Dis. 2012;6, e1831.

24. Huang K-Y, Chen Y-YM, Fang Y-K, Cheng W-H, Cheng C-C, Chen Y-C, et al. Adaptive responses to glucose restriction enhance cell survival, antioxidant capability, and autophagy of the protozoan parasite Trichomonas vaginalis. Biochim Biophys Acta. 1840;2014:53-64.

25. Aurrecoechea C, Brestelli J, Brunk BP, Carlton JM, Dommer J, Fischer S, et al. GiardiaDB and TrichDB: integrated genomic resources for the eukaryotic protist pathogens Giardia lamblia and Trichomonas vaginalis. Nucleic Acids Res. 2009;37:D526-30.

26. Mallo N, Lamas J, Leiro JM. Hydrogenosome metabolism is the key target for antiparasitic activity of resveratrol against Trichomonas vaginalis. Antimicrob Agents CH. 2013;57:2476-84

27. Lehker MW, Alderete JF. Iron regulates growth of Trichomonas vaginalis and the expression of immunogenic trichomonad proteins. Mol Microbiol. 1992;6:123-32.

28. De Jesus JB, Cuervo P, Junqueira M, Britto C, Silva-Filho FCE, Soares MJ, et al. A further proteomic study on the effect of iron in the human pathogen Trichomonas vaginalis. Proteomics. 2007;7:1961-72.

29. Boutrin M-C, Wang C, Aruni W, Li X, Fletcher HM. Nitric oxide stress resistance in Porphyromonas gingivalis is mediated by a putative hydroxylamine reductase. J Bacteriol. 2012;194:1582-92.

30. Justino MC, Baptista JM, Saraiva LM. Di-iron proteins of the Ric family are involved in iron-sulfur cluster repair. Biometals. 2009;22:99-108.

31. Yurgel SN, Rice J, Kahn ML. Transcriptome analysis of the role of GlnD/GlnBK in nitrogen stress adaptation by Sinorhizobium meliloti Rm1021. PLoS One. 2013;8, e58028.

32. Harris KM, Goldberg B, Biagini GA, Lloyd D. Trichomonas vaginalis and Giardia intestinalis produce nitric oxide and display NO-synthase activity. J Eukaryot Microbiol. 2006;53:S182-3.

33. Simon A, Karbach S, Habermeier A, Closs El. Decoding the substrate supply to human neuronal nitric oxide synthase. PLoS One. 2013;8, e67707.

34. Nisoli E, Carruba MO. Nitric oxide and mitochondrial biogenesis. J Cell Sci. 2006;119:2855-62.

35. Ly JD, Grubb DR, Lawen A. The mitochondrial membrane potential $(\Delta \psi \mathrm{m})$ in apoptosis; an update. Apoptosis. 2003;8:115-28.

36. Vanácová S, Rasoloson D, Rázga J, Hrdy I, Kulda J, Tachezy J. Iron-induced changes in pyruvate metabolism of Tritrichomonas foetus and involvement of iron in expression of hydrogenosomal proteins. Microbiology. 2001;147:53-62.

37. Pacheco-Yepez J, Jarillo-Luna RA, Gutierrez-Meza M, Abarca-Rojano E, Larsen BA, Campos-Rodriguez R. Peroxynitrite and peroxiredoxin in the pathogenesis of experimental amebic liver abscess. Biomed Res Int 2014;2014:324230.

38. Pantopoulos K, Hentze MW. Nitric oxide signaling to iron-regulatory protein: direct control of ferritin mRNA translation and transferrin receptor mRNA stability in transfected fibroblasts. Proc Natl Acad Sci U S A. 1995;92:1267-71.

39. Graziano M, Lamattina L. Nitric oxide accumulation is required for molecular and physiological responses to iron deficiency in tomato roots. Plant J. 2007:52:949-60.

40. Sun B, Jing Y, Chen K, Song L, Chen F, Zhang L. Protective effect of nitric oxide on iron deficiency-induced oxidative stress in maize (Zea mays) J Plant Physiol. 2007;164:536-43.

41. Reif DW, Simmons RD. Nitric oxide mediates iron release from ferritin Arch Biochem Biophys. 1990;283:537-41.

42. Maupin-Furlow J. Proteasomes and protein conjugation across domains of life. Nat Rev Microbiol. 2012;10:100-11.

43. Suraweera A, Münch C, Hanssum A, Bertolotti A. Failure of amino acid homeostasis causes cell death following proteasome inhibition. Mol Cell. 2012;48:242-53.

44. Yarlett N, Martinez MP, Moharrami MA, Tachezy J. The contribution of the arginine dihydrolase pathway to energy metabolism by Trichomonas vaginalis. Mol Biochem Parasitol. 1996;78:117-25.

45. Lucas KA, Pitari GM, Kazerounian S, Ruiz-Stewart I, Park J, Schulz S, et al. Guanylyl cyclases and signaling by cyclic GMP. Pharmacol Rev. 2000;52:375-414.
46. Saini AS, Shenoy GN, Rath S, Bal V, George A. Inducible nitric oxide synthase is a major intermediate in signaling pathways for the survival of plasma cells. Nat Immunol. 2014;15:275-82.

47. Diaz CA, Allocco J, Powles MA, Yeung L, Donald RGK, Anderson JW, et al Characterization of Plasmodium falciparum cGMP-dependent protein kinase (PfPKG): antiparasitic activity of a PKG inhibitor. Mol Biochem Parasitol. 2006;146:78-88.

48. Donald RGK, Allocco J, Singh SB, Nare B, Salowe SP, Wiltsie J, et al. Toxoplasma gondii cyclic GMP-dependent kinase: chemotherapeutic targeting of an essential parasite protein kinase. Eukaryot Cell. 2002;1:317-28.

\section{Submit your next manuscript to BioMed Central and take full advantage of:}

- Convenient online submission

- Thorough peer review

- No space constraints or color figure charges

- Immediate publication on acceptance

- Inclusion in PubMed, CAS, Scopus and Google Scholar

- Research which is freely available for redistribution 